\title{
Measurement and Research Using the Big Five, HEXACO, and Narrow Traits: A Primer for Researchers and Practitioners
}

\author{
Jeromy Anglim, Peter O'Connor ${ }^{1}$
}

\begin{abstract}
Objective. Personality traits influence human behaviour across a broad range of situations and are consequently relevant to many theoretical and applied disciplines. In this perspective piece, we provide an overview of the logic underpinning personality measurement and review major personality taxonomies. We provide an extensive set of recommendations for researchers and practitioners on when and how to use measures of personality traits. Method. We overview a range of taxonomic representations of personality structure focusing particularly on hierarchical representations and five and six factor models such as the Big Five and HEXACO models. We review the various strengths and weaknesses of each approach. Results. The review outlines the major reasons for the dominance of the Big Five model, and suggests it is a good descriptive framework for studying personality in general. However we suggest that researchers and practitioners also consider alternative taxonomic personality representations such as the HEXACO. We provide a range of scenarios whereby alternative frameworks will be more appropriate than the Big Five and offer recommendations both for choosing measures in general and for implementing studies examining personality facets. Conclusions. Whilst the Big Five represents an excellent general personality framework that is appropriate across multiple situations, researchers and practitioners should be aware of alternative measures and utilise them where appropriate.
\end{abstract}

Keywords: Big Five, personality facets, HEXACO, narrow traits, personality traits

${ }^{1}$ Anglim, J., \& O'Connor, P. (2018). Measurement and Research Using the Big Five, HEXACO, and Narrow Traits: A Primer for Researchers and Practitioners. Australian Journal of Psychology.

http://dx.doi.org/10.1111/ajpy.12202

Please see the doi to publisher's copy of record and check for updated volume and page numbers.

Jeromy Anglim, School of Psychology, Deakin University, Geelong, Australia; Peter O'Connor, School of Management, Queensland University of Technology, Queensland, Australia. Correspondence concerning this article should be addressed to Jeromy Anglim, School of Psychology, Deakin University, Locked Bag 20000, Geelong, 3220 Australia. Email: jeromy.anglim@ deakin.edu.au 


\section{Key Points}

\section{What is already known about this topic?}

- The Big Five is the dominant personality trait taxonomy

- The HEXACO model is an alternative taxonomy of personality traits

- Personality traits can be organised hierarchically into broad and narrow traits

\section{What the topic adds?}

- The nature and scope of the Big Five are commonly misunderstood

- Researchers should consider using the HEXACO model when investigating morally-relevant behaviours.

- The paper provides a set of practical recommendations for selecting and using personality questionnaires.

\section{Introduction}

Personality traits can be defined as relatively stable patterns of thoughts, feelings and behaviours on which people differ (McCrae \& Costa, 1995). They are frequently used by researchers to describe and classify individuals (e.g. optimistic, ambitious, aggressive, etc.) often in order to explain and predict some external phenomena of interest (e.g. academic performance, Poropat, 2009). Personality traits are termed 'narrow' when they relate to a specific set of tendencies, and to more concrete behaviours (e.g. talkativeness, risk-taking), and are termed 'broad' when they relate to a more general set of tendencies, and to more highly abstracted aspects of behaviour and experience (e.g. extraversion). Since there are many stable aspects of human behaviour, there are also many potential traits researchers could propose to capture these aspects. Indeed early research on personality was characterised by a proliferation of such traits and associated measurement tools, with researchers often proposing new, or alternative versions of existing traits in order to determine or 'discover' the trait underlying some phenomena of interest (e.g., leadership, see Bass \& Stogdill, 1990). By the early 1990s, measures had been developed for hundreds of personality traits, many of which were developed by different research teams and varied only slightly from each other. This proliferation of traits in the absence of an organising framework resulted in a fragmented personality trait literature, where it was difficult to synthesise research findings.

Partially in response to this proliferation, the Big Five emerged in the early 1990s as a unifying taxonomy of personality traits. The Big Five was influential and necessary in personality psychology because it provided five broad, empirically derived traits that collectively accounted for the major dimensions upon which individuals differ: extraversion, agreeableness, 
conscientiousness, neuroticism, and intellect/openness (Digman, 1990; Goldberg, 1990). It also served as an organising framework for the proliferation of traits because most of these traits aligned with one or more of the Big Five dimensions. This supported theoretical development and meta-analytic synthesis. A further advantage of the Big Five was its predictive power; because it arguably captured the five most fundamental dimensions of personality, it was able to predict a range of important outcomes including academic performance (Poropat, 2009), job performance (Barrick \& Mount, 1991; Hurtz \& Donovan, 2000), counterproductive work behaviour (Dalal, 2005), wellbeing (Anglim \& Grant, 2016; Steel, Schmidt, \& Shultz, 2008; Sun, Kaufman, \& Smillie, 2017), and mortality (Bogg \& Roberts, 2004).

However, the success of the Big Five taxonomy has coincided with a growing misunderstanding of what the Big Five represents and how it is best used. One common misconception is that the Big Five is synonymous with personality. Indeed as noted by DeYoung (2015), it is common for researchers to claim that they "measured" or "controlled" for personality by administering a Big Five questionnaire. Although the Big Five represents the major dimensions of personality, positing that it is personality would reduce other trait constructs (e.g. self-esteem, need for cognition, perceived social power, subjective well-being, etc.) to "somehow not measures of personality" (DeYoung, 2015, p. 36), which is clearly not the case. More generally, the Big Five was not intended to represent all meaningful variance in personality traits, but instead the Big Five represents one level of breadth in a comprehensive hierarchical representation of personality.

Second, the rise in popularity of short-form measures of personality (e.g., 1, 2, 3, and 4 items per scale) has led to substantial under estimation of the predictive validity of the Big Five (for a review, see Credé, Harms, Niehorster, \& GayeValentine, 2012). The Big Five represent broad dimensions of personality and Big Five questionnaires should consequently sample a broad range of behaviours. Using short measures with poor reliability not only leads to underestimates in bivariate correlations but also leads to inappropriate conclusions when controlling for the Big Five in studies seeking to establish the incremental validity of other instruments. Although commonly done, it is inappropriate to claim support for the incremental validity of a new measure over the Big Five when the Big Five is measured using a short-form. Although technically the new measure will have incremental validity over the short-form measure of the Big Five, this may not have been the case had a longer, more reliable measure of the Big Five been used. 
Third, researchers have often over-interpreted the original goals of the Big Five taxonomy. As detailed later, the Big Five was developed to describe and classify individuals in terms of the major dimensions discriminating between people. Multiple sources of evidence indicate that the Big Five is an excellent framework for doing this. The Big Five was not developed to explain why people differ and consequently is not a theory of personality per se. Similarly, it does not provide a within-person account of personality (i.e. describing predictable patterns of within-person variation over time) but rather a between person account of personality (i.e. describing between person variation in personality traits). Consequently critiques on the Big Five based on a lack of underlying theory are unfounded; the goal of Big Five researchers has primarily been to describe rather than explain variation in personality. Whilst the study of within-person personality systems and between-person trait structure are not independent, optimal taxonomic structure is primarily an empirical question, and should be guided more by utility, comprehensiveness and reliability than consistency with a theoretical perspective at this point (see Cervone, 2005; Collins, Jackson, Walker, O’Connor, \& Gardiner, 2017). Although theoretical models have been developed based on the Big Five (e.g., the Cybernetic Big Five, DeYoung, 2015), these represent significant extensions on the Big Five and should not be considered as equivalent to the Big Five.

The current article represents a primer for researchers and practitioners. It seeks to provide a user-friendly account of the methods researchers have used to identify major personality traits and explains how the Big Five emerged as the dominant descriptive model of personality. It also provides an overview of an alternative popular personality taxonomy (the HEXACO model) and provides guidance on choosing between this model and the Big Five. It also reviews fundamental themes in the personality literature related to personality as a hierarchy and the relative pros and cons of measuring narrow traits. In particular, the current article shows how broad traits such as the Big Five represent but one of several levels of the personality trait hierarchy. It then provides guidance to researchers who seek to measure personality in both research and applied contexts.

\section{Trait Taxonomies and The Big Five}

What is a generalizable taxonomy of personality traits? The term 'taxonomy' is generally used to describe the scientific endeavour to classify and describe. From this perspective therefore, a generalizable taxonomy of personality is one that can adequately classify and describe a broad set of individuals in terms of a well-defined set of criteria. Saucier and Srivastava (2015) proposed eight criteria for judging the effectiveness of a possible 
taxonomy: social importance, predictive power, comprehensiveness, reliability and cross-time stability, generalizability across data collection formats, causal basis, and a theory. Others have specified fewer criteria for determining optimal taxonomies and place less emphasis on theory; for example, Revelle (1995) specified that many taxonomists focus on parsimony, replicability and usefulness when determining descriptive taxonomies. Consistent with Revelle (1995) and our earlier clarification of the goals of the Big Five taxonomy, we do not regard a causal theory as necessary for defining a taxonomy of personality traits.

The search for a consistent set of broad traits has been heavily influenced by what is known as the lexical hypothesis. It posits that the major dimensions of personality are encoded in human language, with the more salient characteristics encoded as single words. Early lexical studies used dictionary searches to identify and categorise personality-descriptive terms (Allport \& Odbert, 1936; Thurstone, 1934). The set of terms were reduced from thousands to a smaller set using synonyms and applying various statistical techniques such as factor analysis. Although these datasets provided initial support for a Big Five (e.g., Thurstone, 1934), the most influential basis for a Big Five came from Goldberg $(1981,1990)$ who conducted factor analyses on comprehensive sets of trait adjectives. Over multiple studies Goldberg found support for the "Big Five" factors of personality. Strong support for a five-factor model was also obtained in various studies by McCrae and Costa (1987) who repeatedly found five dimensions across instruments and observers (self and peerreports). On balance, lexical studies along with studies using large questionnaires tend to support the idea that personality can broadly be described using the Big Five dimensions of personality (Condon, 2017; Costa \& McCrae, 1995; Markon, Krueger, \& Watson, 2005).

\section{An Alternative Taxonomy: The HEXACO}

Although many lexical studies provide support for the Big Five, several more recent studies have provided support for alternative personality taxonomies (for reviews and important studies, see Ashton et al., 2004; De Raad, Barelds, Levert, et al., 2010; De Raad \& Mlacic, 2017; De Raad, Perugini, \& Szirmák, 1997; Peabody \& De Raad, 2002; Saucier \& Goldberg, 2001; Saucier \& Srivastava, 2015). In particular, several lexical studies in languages other than English that have used different adjective inclusion criteria have found support for six factors (Ashton \& Lee, 2001). The most well-known and influential of these six factor taxonomies is known as the 'HEXACO' model (Ashton, Lee, \& de Vries, 2014; Ashton et al., 2004). HEXACO is an 
acronym for six broad traits: honesty-humility, emotionality, extraversion, agreeableness, conscientiousness, and openness. The biggest difference between the Big Five and HEXACO model is the addition of the honesty-humility factor, represented by the facets of sincerity, fairness, greed avoidance, and modesty. Strictly speaking however, this factor does not represent an 'addition' to the Big Five, but rather represents the repartitioning of the variance of Big Five neuroticism and agreeableness into HEXACO agreeableness, emotionality, and honesty-humility.

Ashton and Lee (2001) noted that a seventh factor in these lexical studies led to small and inconsistent factors. They suggested that it is the space formed by the extracted dimensions rather than the particular rotation selected that is most important. Thus, from a predictive validity perspective, it is better to err on the side of one more factor than one less. In reviewing the literature, Saucier, Hampson, Goldberg, and Hampson (2000) suggested that models with fewer factors (e.g., 1, 2, or 3) may be more cross-culturally replicable. However, Ashton and Lee (2001) suggested that while emotionality, extraversion, agreeableness and conscientiousness are more universal, openness and honestyhumility are important, and that representing them as broad traits is an advantage.

Although the HEXACO model is receiving growing support, the necessity of a sixth broad trait, as well as the nature of this trait are subjects of current debate. First, other prominent six factor inventories including the Hogan Personality Inventory (Hogan \& Hogan, 1992), the Six Factor Personality Questionnaire (Jackson, Paunonen, \& Tremblay, 2000), and Saucier's Big Six (Saucier, 2009) have not converged on the same sixth factor in the same way that has broadly occurred with the Big Five (De Raad, Barelds, Mlačić, et al., 2010). Second, while several lexical studies have broadly supported the HEXACO model, the support is far from universal. Third, Saucier and Ostendorf (1999) have argued that any broad trait in addition to the Big Five will correlate highly with one of the existing Big Five, and honesty-humility correlates relatively highly with agreeableness ( $r=.67$ in Gaughan, Miller, \& Lynam, 2012). Honesty-humility also correlates reasonably well with straightforwardness and modesty facets of agreeableness in the NEO-PI-R, suggesting that a comprehensive facet-level model using the Big Five may provide similar predictive benefits (Costa \& McCrae, 1995; Gaughan et al., 2012). Fourth, Barford, Zhao, and Smillie (2015) mapped the Big Five and HEXACXO traits to a common space provided by the interpersonal circumplex, and found that honesty-humility occupied a fairly similar space to the politeness aspect of B5 agreeableness. Finally, Condon (2017) assessed the covariance structure of approximately 700 statement- 
based personality test items drawn from the most commonly used public-domain personality measures (including an approximately equal representation of items from prominent five and six-factor measures). While he found support for a large number of narrow traits, factor analysis suggested five broad traits, and when six factors were extracted, the sixth factor did not correspond to honesty-humility.

Regardless of whether five or six factors are required to adequately describe personality, there are numerous practical benefits to using a six factor solution. Honesty-humility is a good predictor of a range of important outcomes over and above the Big Five. This is particularly true for unethical, manipulative, and deceptive behaviour (e.g., Jonason \& O'Connor, 2017; Lee, Ashton, Morrison, Cordery, \& Dunlop, 2008; Lee, Gizzarone, \& Ashton, 2003) as well as prosocial behaviour (e.g., Zhao \& Smillie, 2015). When honesty-humility is reversed, it is almost synonymous with measures of the Dark Triad (Aghababaei, Mohammadtabar, \& Saffarinia, 2014; Lee \& Ashton, 2014). Thus, the inclusion of honesty-humility aligns with recent interest in dark personality (Smith, Hill, Wallace, Recendes, \& Judge, 2018). The HEXACO model may also be particularly useful in employee selection settings (Anglim, Morse, De Vries, MacCann, \& Marty, 2017; Hough \& Connelly, 2013) where organisations seek to identify job applicants who are more likely to engage in bullying, theft, and other counterproductive work behaviours,.

\section{Personality Traits as a Hierarchy}

While lexical studies have focused primarily on the number and nature of broad traits, factor analyses of large item pools also provide evidence that traits can be represented in terms of a nested hierarchy (see Table 1 for popular examples). Hierarchical models of personality typically consist of one or more broad traits at the highest levels and progressively narrower and more numerous traits at lower levels. In these models a set of broad traits are each composed of several facets which help to define the broad trait and provide a more nuanced perspective of personality. Many modern comprehensive personality tests are nested hierarchies; prominent examples include the 30 facets and 5 domains of the NEO-PI-R (Costa \& MacCrae, 1992), the 25 facets and 6 domains of the HEXACO-PI-R (Ashton, Lee, et al., 2014), De Young's 10 aspects of the Big Five (DeYoung, Quilty, \& Peterson, 2007) and the 15 facets and 5 factors of the BFI-2 (Soto \& John, 2017); see also the 25 facets and 5 factors of the PID-5 for assessing 'abnormal'/clinical personality (Markon, Quilty, Bagby, \& Krueger, 2013). 
Table 1

A Selection of Popular Hierarchical Models of Personality Traits

Model : Broad Trait: Narrow Traits

NEO-PI-R (Costa \& MacCrae, 1992)

1. Neuroticism: Anxiety, Hostility, Depression, Self-consciousness, Impulsiveness, Vulnerability to Stress

2. Extraversion: Warmth, Gregariousness, Assertiveness, Activity, Excitement Seeking, Positive Emotion

3. Openness to Experience: Fantasy, Aesthetics, Feelings, Actions, Ideas, Values

4. Agreeableness: Trust, Straightforwardness, Altruism, Compliance, Modesty, Tendermindedness

5. Conscientiousness: Competence, Order, Dutifulness, Achievement Striving, Self-Discipline, Deliberation

Big Five Aspects (DeYoung et al., 2007)

1. Neuroticism: Withdrawal, Volatility

2. Extraversion: Enthusiasm, Assertiveness

3. Openness: Openness/Creativity, Intellect

4. Agreeableness: Politeness, Compassion

5. Conscientiousness: Orderliness, Industriousness

BFI-2 (Soto \& John, 2017)

1. Extraversion: Sociability, Assertiveness, Energy Level

2. Agreeableness: Compassion, Respectfulness, Trust

3. Conscientiousness: Order, Self-Discipline, Dutifulness

4. Negative Emotionality: Anxiety, Depression, Emotional Volatility

5. Open-Mindedness: Intellectual Curiosity, Aesthetic Sensitivity, Creative Imagination

HEXACO-PI-R (Ashton, Lee, et al., 2014)

1. Honesty-Humility: Sincerity, Fairness, Geed Avoidance, Modesty

2. Emotionality: Fearfulness, Anxiety, Dependence, Sentimentality

3. Extraversion: Social Self-Esteem, Social Boldness, Sociability,

Liveliness

4. Agreeableness: Forgiveness, Gentleness, Flexibility, Patience

5. Conscientiousness: Organization, Diligence, Perfectionism, Prudence

6. Openness to Experience: Aesthetic Appreciation, Inquisitiveness, Creativity, Unconventionality

Interstitial Traits: Altruism

Note. Broad traits in bold, narrow traits follow the colon. HEXACO has the interstitial trait of altruism that spans multiple broad traits.

Long-form questionnaires designed to measure the Big Five have typically operationalised the Big Five traits as existing at the highest level of the hierarchy, with narrower traits or 'facets' at lower levels (Anglim \& Grant, 2014; Anglim \& Grant, 2016; Ashton, Lee, Goldberg, \& De Vries, 2009; Chang, Connelly, \& Geeza, 2012; Costa \& McCrae, 1992; Davies, Connelly, Ones, \& Birkland, 2015). Extending the two-level division of domains and facets, higher, intermediate, and lower hierarchical levels have also 
been proposed. Researchers have posited one and two factor higher-order models of personality suggesting that the Big Five may not be the broadest meaningful level of personality analysis (Anusic, Schimmack, Pinkus, \& Lockwood, 2009; Digman, 1997; Musek, 2007; Veselka et al., 2009). Notably, a single, broad factor (termed the General Factor of Personality; GFP) has been proposed to account for meaningful trait variance at the highest level (e.g., Musek, 2007), although see various critical perspectives (Davies et al., 2015; De Vries, 2011; Revelle \& Wilt, 2013). There is also evidence for two factors termed 'stability' and 'plasticity' existing at the highest level of analysis (see DeYoung, 2006, 2015). DeYoung (2015) has proposed ten personality aspects (two for each of the Big Five) as traits of intermediate breadth between domains and facets. Finally, other researchers further decompose facets into nuances commonly operationalised as items (McCrae, 2015; Mõttus, Kandler, Bleidorn, Riemann, \& McCrae, 2017; Mõttus, McCrae, Allik, \& Realo, 2014). In general, while other structural representations have been proposed such as bifactor models (Anglim, Morse, et al., 2017; Biderman, Nguyen, Cunningham, \& Ghorbani, 2011; Chen, Watson, Biderman, \& Ghorbani, 2016; Klehe et al., 2012), lists (Loehlin \& Goldberg, 2014), network models (Cramer et al., 2012; Guillaume-Hanes, Morse, \& Funder, 2012; Wilt, Condon, Brown-Riddell, \& Revelle, 2012), and circumplex models (Barford et al., 2015; DeYoung, Weisberg, Quilty, \& Peterson, 2013; Hofstee, De Raad, \& Goldberg, 1992; Morris, Burns, \& Periard, 2015), it seems that the organisation of traits into a hierarchy is a useful tool for conceptualising and organising personality traits, even if the common division into two-levels (domains and facets) is merely a conceptual convenience (Costa \& McCrae, 1995).

\section{The Utility of Narrow Traits}

The utility of narrow traits has been actively debated (Anglim \& Grant, 2014; Anglim \& Grant, 2016; Ashton, 1998; Ashton, Jackson, Paunonen, Helmes, \& Rothstein, 1995; Ashton, Paunonen, \& Lee, 2014; Christiansen \& Robie, 2011; O'Neill, Paunonen, Christiansen, \& Tett, 2013; Ones \& Viswesvaran, 1996; Paunonen, 1998; Paunonen \& Ashton, 2001; Paunonen, Haddock, Forsterling, \& Keinonen, 2003; Paunonen, Rothstein, \& Jackson, 1999; Salgado, Moscoso, \& Berges, 2013). Compared to the broad Big Five, narrow traits generally offer enhanced predictive validity. There is good empirical evidence that, collectively, narrow traits are better predictors of outcomes than broad traits (e.g., Anglim \& Grant, 2014; Anglim \& Grant, 2016) particularly when the outcome is narrow (Dudley, Orvis, Lebiecki, \& Cortina, 2006). A common finding seems to be a modest but meaningful incremental prediction (Anglim, Bozic, Little, \& Lievens, 2018; 
Anglim \& Grant, 2014; Anglim \& Grant, 2016; Anglim, Knowles, Dunlop, \& Marty, 2017; Sun et al., 2017). Statistically these findings are not surprising considering that factor analytic solutions require trade-offs between total variance explained and parsimony, such that reduced factor solutions will always account for less variation in original dimensions than non-reduced solutions. There is also increasing interest in using large samples and many indicators combined with machine learning algorithms to optimize prediction in applied settings (Bleidorn, Hopwood, \& Wright, 2017; Chamorro-Premuzic, Akhtar, Winsborough, \& Sherman, 2017).

Debates about five versus six factors notwithstanding, compared to broad traits, there is less agreement about the number and nature of narrow traits (Woods \& Anderson, 2016). In general, compared to broad traits, facets in popular frameworks have tended to be derived more by rational means. Facets have been drawn from the conceptual diversity of adjectives used to define broad traits in lexical studies (Ashton et al., 2004), as well as searches of the literature for relevant psychological constructs (Costa, McCrae, \& Dye, 1991). Such frameworks are then typically reinforced by factor analytic evidence. Instead of being definitive, such frameworks often seek to cover the field of important narrow traits. While theoretical development on the criteria by which to judge facet-level frameworks is still needed. some progress is being made. The 10 aspects (DeYoung et al., 2007) and the 15 facets and 5 factors of the BFI-2 (Soto \& John, 2017) contain considerable overlap. One interesting line of work by Condon (2017) suggests that something like a lexical study can be performed using large item pools. While getting participants to complete 1000s of items is not readily possible, it is possible to get thousands of participants to do a smaller random samples of items, and then generate covariance matrices that can be used in latent variable models. This is the basis of the Synthetic Aperture Personality Assessment Project (Revelle et al., 2016; Wilt, Condon, \& Revelle, 2012). This offers an intriguing approach for obtaining an empirically derived structure of narrow traits.

\section{Recommendations for Researchers}

With regards to the decision of whether to use the Big Five or the HEXACO model, we offer the following suggestions. Both frameworks offer a strong basis for examining individual differences in other traits. While popular, there is still ample potential to do novel research using the HEXACO framework. In particular, if the outcome of interest includes morally relevant behaviours, then inclusion of the honesty-humility factor either as a single scale in addition to a measure of the Big Five or using the HEXACO model will often yield novel insights and improved 
prediction. In general, short-form measures of personality should be avoided (i.e. measures with one to four items per scale). If researchers want to use a good relatively short measure, there are a range of good measures with between 8 and 12 items per factor. The International Personality Item Pool (IPIP) measures (Goldberg et al., 2006) including Goldberg's Big-Five Factor Markers and measures based on the NEO-PI-R and other instruments, have the advantage of being explicitly in the public domain, which facilitates open science (http://ipip.ori.org/).

In addition to decisions about measurement of broad traits, researchers and practitioners also need to decide whether to measure and analyse narrow traits, and if so, which framework to use. In general, when sample size is sufficiently large, research that combines broad domain and facet-level measurement provide a more complete picture of personality. Predictive validity and explanatory power are both improved. However, research applying facet-level frameworks involves several additional challenges related to design, analysis, and interpretation. Many of these issues relate to balancing parsimony and completeness. While research purely concerned with predictive validity can use a range of different personality measures often in an attempt to optimise the trade-off between length and validity, the following recommendations pertain to studies seeking to contribute to a comprehensive understanding of how personality relate to other variables.

To encourage more research that rigorously maps personality facets onto outcomes of interest, we offer the following suggestions regarding designing studies involving facet-level measurement (for further review, see Anglim \& Grant, 2014). First, we recommend using a measure that attempts to provide a comprehensive factor-facet framework using a questionnaire format with the full set of items (typically 8 or more items per facet). Examples include the 240 item NEO-PI-R, the 200 item HEXACO-PI-R, or the IPIP equivalents such as the 300 item IPIPNEO (Goldberg, 1999). If these long questionnaires are impractical, then it is often best to focus interpretation only on broad traits, although the BFI-2 (60 items) and the Big Five aspects scale (100 items) are also good options. Reliable facet-level measurement is essential, especially when researchers are trying to reliably estimate the unique variance associated with a facet and not just the variance associated with the corresponding broad trait. Second, avoid simply measuring the facets of one factor (e.g., only the six facets of conscientiousness). Facets may overlap with unmeasured broad traits and may inaccurately represent the incremental prediction of a particular facet. Third, Sample size is critically important. The large number of predictors in regression 
models, and the multiplicities involved in comparing facet-level correlations mean that large sample sizes are essential. If the sample size is less than 200, then comprehensive facet-level analyses should generally be avoided, and it may be better to focus on broad traits. In particular, the subtle comparisons of interest benefit greatly from large samples: 500 is good, 1000 is better. Greater understanding of the trade-off between sample size and precision can be assessed using simulations (see Anglim \& Grant, 2014).

With regards to the analysis of facet-level personality data, we offer the following suggestions. First, when comparing prediction of models involving facets with those involving factors, it is important to quantify prediction using a statistical method that appropriately penalises for the larger number of predictors in facetlevel regression models. An appropriate way to do this is to use adjusted r-squared (or adjusted multiple r). Simply using raw rsquared will exaggerate the incremental prediction of facets. In contrast, adjusted r-squared is an unbiased estimate of population variance explained that corrects for the tendency for models with more predictors to account for additional noise in the data, an effect which is amplified with smaller sample sizes.

Second, it is important to have an understanding of standard errors and confidence intervals on the incremental variance explained. The personalityfacets package in $\mathrm{R}$ provides on means of obtaining bootstrap confidence intervals on incremental variance explained (Anglim \& Grant, 2014). In particular, if the aim is to get relatively precise estimates of incremental variance explained, then the required sample size may need to be around 1000 or more.

Third, in addition to reporting facet-criterion correlations in raw form, it is insightful to examine the unique correlation after partialling out the effect of broad traits. This is beneficial because it presents a more parsimonious picture of which facets add information. For example, Anglim et al. (2018) found some evidence for all facets of conscientiousness correlating with academic grades in medical school, but it was mostly selfdiscipline and achievement striving that showed beneficial incremental prediction. The two main approaches to assessing incremental value are residualized facets and bifactor modelling. Residualized facets is the most extreme in that it assigns all common variance to the broad traits. In contrast, the bifactor model (Chen, Hayes, Carver, Laurenceau, \& Zhang, 2012; McAbee, Oswald, \& Connelly, 2014; Perera, Izadikhah, O’Connor, \& McIlveen, 2016) distributes common variance between factors and facets. The bifactor model also provides a way of separating evaluative variance from more descriptive trait variance (Anglim, 
Morse, et al., 2017). Fourth, due to the many relationships being examined, it is important to use a more stringent alpha when assessing facet-criteria relationships whether they be zero-order, bifactor coefficients, or residualized correlations. For example, if working with a 30 facet framework, an alpha of .001 is a good option, as it roughly corresponds to .05 alpha divided by 30 . A less stringent alpha could potentially be adopted where a small number of specific facet-criteria relationships are preregistered. Various examples of the above approach include studies of well-being (Anglim \& Grant, 2016), values (Anglim, Knowles, et al., 2017), and Type D personality (Horwood, Anglim, \& Tooley, 2015).

Finally, further considerations are required if the aim of the analysis is applied prediction. For example, in employee selection contexts, the aim is often to use a regression equation to predict employee behaviour. Whereas adjusted r-squared provides an estimate of the population prediction of a set of predictors, this estimate is always larger than the estimated cross-validation prediction (Anglim \& Grant, 2014). This is because, adjusted rsquare is predicated on the regression equation being known, whereas in practice, the regression equation must be specified based on only partial knowledge of the true regression equation. In general, this gap between adjusted r-squared and cross-validated rsquared declines as sample size increases. The gap is also smaller when there are fewer predictors. A consequence of this is that if the validation sample is only moderate (e.g., 200 to 500), then it may be the case that broad traits yield equivalent cross-validated $\mathrm{r}$ squared even when facets provide incremental prediction using the population regression equation. This point is amplified further when an item-level nuance approach to prediction is adopted.

We also caution test publishers against, and advise test consumers to be wary of, practices which may exaggerate the predictive validity of personality tests. Examining correlations between a large number of facets or items and a criteria and then using the best correlating predictors in a regression model using the same data is particularly problematic and can dramatically exaggerate prediction. Instead, either all potential predictors should be included in the model and adjusted r-square or cross-validated rsquared should be used, or a separate validation sample should be sought. A range of other problematic practices include selective reporting of studies and selective reporting of outcome measures. In many applied domains, when using objective criteria, correlations with personality may be modest, and surprisingly large samples, especially in applied settings, may be required to yield good statistical power. In particular, it is the raw unadjusted correlations from meta-analyses that should inform power 
analyses, rather than the adjusted theoretical correlations that might be obtained were measurement perfect.

Finally, we encourage researchers to adopt open science practices in order to facilitate research synthesis in research involving personality. Several personality journals now encourage the sharing of raw data, materials, and data analysis scripts (e.g., Back, 2018) using repositories such as the Open Science Framework. Examples of research on hierarchical models of personality with raw data and data analysis scripts available include Anglim, Knowles, et al. (2017); Anglim, Morse, et al. (2017); Horwood et al. (2015). The IPIP is also a great example of a set of items and measures with open licences (Goldberg, 1999). This facilitates the deeper assessment of structural relations, and synthesis across measures is facilitated. In particular, more research implementing open science is needed to tease out item, domain, and facet-level mappings between scales and the novelty of various constructs.

\section{Conclusion}

To conclude, we reiterate that the Big Five model represents a good descriptive framework for studying personality in general. We suggest that researchers and practitioners use the Big Five when seeking to measure or control for a relatively complete set of broad personality traits. However we also suggest that researchers and practitioners consider alternative taxonomic personality representations such as the HEXACO, particularly when studying morally relevant behaviours. Where possible, we advise that researchers and practitioners use long measures of the Big Five or HEXACO in order to obtain measures of broad and narrow traits. Where long measures are not practical, we recommend researchers measure only broad traits. Finally, we caution researchers against various practices likely to lead to incorrect conclusions when working with personality data. In particular, researchers should use large sample sizes, interpret adjusted $\mathrm{R}$ squared, use conservative $\mathrm{p}$-values, and cross-validate findings when focusing on applied prediction.

\section{References}

Aghababaei, N., Mohammadtabar, S., \& Saffarinia, M. (2014). Dirty Dozen vs. the $\mathrm{H}$ factor: Comparison of the Dark Triad and Honesty-Humility in prosociality, religiosity, and happiness. Personality and Individual Differences, 67, 6-10.

Allport, G. W., \& Odbert, H. S. (1936). Trait-names: A psycho-lexical study. Psychological monographs, 47(1), i-171.

Anglim, J., Bozic, S., Little, J., \& Lievens, F. (2018). Response distortion on personality tests in applicants: comparing high-stakes to low-stakes medical settings. Advances in Health Sciences Education, Advanced Online Access. 
Anglim, J., \& Grant, S. L. (2014). Incremental criterion prediction of personality facets over factors: Obtaining unbiased estimates and confidence intervals. Journal of Research in Personality, 53, 148-157.

Anglim, J., \& Grant, S. L. (2016). Predicting psychological and subjective wellbeing from personality: Incremental prediction from 30 facets over the big 5. Journal of Happiness Studies, 17, 59-80.

Anglim, J., Knowles, E. R., Dunlop, P. D., \& Marty, A. (2017). HEXACO Personality and Schwartz's Personal Values: A Facet-Level Analysis. Journal of Research in Personality, 68, 23-31.

Anglim, J., Morse, G., De Vries, R., E., MacCann, C., \& Marty, A. (2017). Comparing Job Applicants to Non-Applicants Using an Item-Level Bifactor Model on the HEXACO Personality Inventory. European Journal of Personality, 31, 669-684.

Anusic, I., Schimmack, U., Pinkus, R. T., \& Lockwood, P. (2009). The nature and structure of correlations among Big Five ratings: The halo-alphabeta model. Journal of Personality and Social Psychology, 97(6), 11421156.

Ashton, M. C. (1998). Personality and job performance: The importance of narrow traits. Journal of Organizational Behavior, 19(3), 289-303.

Ashton, M. C., Jackson, D. N., Paunonen, S. V., Helmes, E., \& Rothstein, M. G. (1995). The criterion validity of broad factor scales versus specific facet scales. Journal of Research in Personality, 29(4), 432-442.

Ashton, M. C., \& Lee, K. (2001). A theoretical basis for the major dimensions of personality. European Journal of Personality, 15(5), 327-353.

Ashton, M. C., Lee, K., \& de Vries, R. E. (2014). The HEXACO HonestyHumility, Agreeableness, and Emotionality factors: A review of research and theory. Personality and Social Psychology Review, 18, 139-152.

Ashton, M. C., Lee, K., Goldberg, L. R., \& De Vries, R. E. (2009). Higher order factors of personality: Do they exist? Personality and Social Psychology Review, 13(2), 79-91.

Ashton, M. C., Lee, K., Perugini, M., Szarota, P., De Vries, R. E., Di Blas, L., . . . De Raad, B. (2004). A six-factor structure of personality-descriptive adjectives: solutions from psycholexical studies in seven languages. Journal of Personality and Social Psychology, 86(2), 356-366.

Ashton, M. C., Paunonen, S. V., \& Lee, K. (2014). On the validity of narrow and broad personality traits: A response to. Personality and Individual Differences, 56, 24-28.

Back, M. D. (2018). Continued Quality, Openness, and Curiosity at the European Journal of Personality. European Journal of Personality, 32(1), 3-5.

Barford, K. A., Zhao, K., \& Smillie, L. D. (2015). Mapping the interpersonal domain: Translating between the Big Five, HEXACO, and Interpersonal Circumplex. Personality and Individual Differences, 86, 232-237.

Barrick, M. R., \& Mount, M. K. (1991). The big five personality dimensions and job performance: a meta-analysis. Personnel Psychology, 44(1), 1-26.

Bass, B. M., \& Stogdill, R. M. (1990). Bass \& Stogdill's handbook of leadership: Theory, research, and managerial applications: Simon and Schuster.

Biderman, M. D., Nguyen, N. T., Cunningham, C. J., \& Ghorbani, N. (2011). The ubiquity of common method variance: The case of the Big Five. Journal of Research in Personality, 45(5), 417-429.

Bleidorn, W., Hopwood, C. J., \& Wright, A. G. (2017). Using big data to advance personality theory. Current Opinion in Behavioral Sciences, $18,79-82$. 
Bogg, T., \& Roberts, B. W. (2004). Conscientiousness and health-related behaviors: a meta-analysis of the leading behavioral contributors to mortality. Psychological Bulletin, 130(6), 887-919.

Cervone, D. (2005). Personality architecture: Within-person structures and processes. Annu. Rev. Psychol., 56, 423-452.

Chamorro-Premuzic, T., Akhtar, R., Winsborough, D., \& Sherman, R. A. (2017). The datafication of talent: how technology is advancing the science of human potential at work. Current Opinion in Behavioral Sciences, 18, 13-16.

Chang, L., Connelly, B. S., \& Geeza, A. A. (2012). Separating method factors and higher order traits of the Big Five: A meta-analytic multitraitmultimethod approach. Journal of Personality and Social Psychology, 102(2), 408-426.

Chen, F. F., Hayes, A., Carver, C. S., Laurenceau, J. P., \& Zhang, Z. (2012). Modeling general and specific variance in multifaceted constructs: A comparison of the bifactor model to other approaches. Journal of Personality, 80(1), 219-251.

Chen, Z., Watson, P., Biderman, M., \& Ghorbani, N. (2016). Investigating the Properties of the General Factor (M) in Bifactor Models Applied to Big Five or HEXACO Data in Terms of Method or Meaning. Imagination, Cognition and Personality, 35, 216-243.

Christiansen, N. D., \& Robie, C. (2011). Further consideration of the use of narrow trait scales. Canadian Journal of Behavioural Science, 43(3), 183-194.

Collins, M. D., Jackson, C. J., Walker, B. R., O’Connor, P. J., \& Gardiner, E. (2017). Integrating the context-appropriate balanced attention model and reinforcement sensitivity theory: Towards a domain-general personality process model. Psychological Bulletin, 143(1), 91-106.

Condon, D. (2017). The SAPA Personality Inventory: An empirically-derived, hierarchically-organized self-report personality assessment model (Draft: March 13th 2017).

Costa, J. P. T., \& McCrae, R. R. (1992). Four ways five factors are basic. Personality and Individual Differences, 13, 653-665.

Costa, P. T., \& MacCrae, R. R. (1992). Revised NEO personality inventory (NEO PI-R) and NEO five-factor inventory (NEO FFI): Professional manual: Psychological Assessment Resources.

Costa, P. T., \& McCrae, R. R. (1995). Domains and facets: Hierarchical personality assessment using the Revised NEO Personality Inventory. Journal of Personality Assessment, 64(1), 21-50.

Costa, P. T., McCrae, R. R., \& Dye, D. A. (1991). Facet scales for agreeableness and conscientiousness: A revision of the NEO Personality Inventory. Personality and Individual Differences, 12(9), 887-898.

Cramer, A. O., Sluis, S., Noordhof, A., Wichers, M., Geschwind, N., Aggen, S. H., . . . Borsboom, D. (2012). Dimensions of normal personality as networks in search of equilibrium: You can't like parties if you don't like people. European Journal of Personality, 26(4), 414-431.

Credé, M., Harms, P., Niehorster, S., \& Gaye-Valentine, A. (2012). An evaluation of the consequences of using short measures of the Big Five personality traits. Journal of Personality and Social Psychology, 102(4), 874.

Dalal, R. S. (2005). A meta-analysis of the relationship between organizational citizenship behavior and counterproductive work behavior. Journal of Applied Psychology, 90(6), 1241-1255.

Davies, S. E., Connelly, B. S., Ones, D. S., \& Birkland, A. S. (2015). The General Factor of Personality: The "Big One," a self-evaluative trait, or 
a methodological gnat that won't go away? Personality and Individual Differences, 81, 13-22.

De Raad, B., Barelds, D. P., Levert, E., Ostendorf, F., Mlačić, B., Blas, L. D., . . . Perugini, M. (2010). Only three factors of personality description are fully replicable across languages: a comparison of 14 trait taxonomies. Journal of Personality and Social Psychology, 98(1), 160-173.

De Raad, B., Barelds, D. P., Mlačić, B., Church, A. T., Katigbak, M. S., Ostendorf, F., . . Szirmák, Z. (2010). Only three personality factors are fully replicable across languages: Reply to Ashton and Lee. Journal of Research in Personality, 44(4), 442-445.

De Raad, B., \& Mlacic, B. (2017). Psycholexical Studies of Personality Structure across Cultures. The Praeger Handbook of Personality Across Cultures [3 volumes], 161.

De Raad, B., Perugini, M., \& Szirmák, Z. (1997). In pursuit of a cross-lingual reference structure of personality traits: Comparisons among five languages. European Journal of Personality, 11(3), 167-185.

De Vries, R. E. (2011). No evidence for a general factor of personality in the HEXACO personality inventory. Journal of Research in Personality, 45(2), 229-232.

DeYoung, C. G. (2006). Higher-order factors of the Big Five in a multiinformant sample. Journal of Personality and Social Psychology, 91(6), $1138-1151$.

DeYoung, C. G. (2015). Cybernetic big five theory. Journal of Research in Personality, 56, 33-58.

DeYoung, C. G., Quilty, L. C., \& Peterson, J. B. (2007). Between facets and domains: 10 aspects of the Big Five. Journal of Personality and Social Psychology, 93(5), 880-896.

DeYoung, C. G., Weisberg, Y. J., Quilty, L. C., \& Peterson, J. B. (2013). Unifying the aspects of the Big Five, the interpersonal circumplex, and trait affiliation. Journal of Personality, 81(5), 465-475.

Digman, J. M. (1990). Personality structure: Emergence of the five-factor model. Annual review of psychology, 41(1), 417-440.

Digman, J. M. (1997). Higher-order factors of the Big Five. Journal of Personality and Social Psychology, 73(6), 1246-1256.

Dudley, N. M., Orvis, K. A., Lebiecki, J. E., \& Cortina, J. M. (2006). A metaanalytic investigation of conscientiousness in the prediction of job performance: examining the intercorrelations and the incremental validity of narrow traits. Journal of Applied Psychology, 91(1), 40-57.

Gaughan, E. T., Miller, J. D., \& Lynam, D. R. (2012). Examining the utility of general models of personality in the study of psychopathy: A comparison of the HEXACO-PI-R and NEO PI-R. Journal of personality disorders, 26(4), 513-523.

Goldberg, L. R. (1981). Language and individual differences: The search for universals in personality lexicons. Review of personality and social psychology, 2(1), 141-165.

Goldberg, L. R. (1990). An alternative" description of personality": the big-five factor structure. Journal of Personality and Social Psychology, 59(6), 1216-1229.

Goldberg, L. R. (1999). A broad-bandwidth, public-domain, personality inventory measuring the lower-level facets of several five-factor models. Personality Psychology in Europe, 7, 7-28.

Goldberg, L. R., Johnson, J. A., Eber, H. W., Hogan, R., Ashton, M. C., Cloninger, C. R., \& Gough, H. G. (2006). The international personality item pool and the future of public-domain personality measures. Journal of Research in Personality, 40(1), 84-96. 
Guillaume-Hanes, E., Morse, P., \& Funder, D. (2012). Network models in the organization of personality. European Journal of Personality, 26(4), 439-440.

Hofstee, W. K., De Raad, B., \& Goldberg, L. R. (1992). Integration of the Big Five and circumplex approaches to trait structure. Journal of Personality and Social Psychology, 63(1), 146-163.

Hogan, R., \& Hogan, J. (1992). Hogan Personality Inventory Manual (2nd ed.). Tulsa, OK

: Hogan Assessment Systems.

Horwood, S., Anglim, J., \& Tooley, G. (2015). Type D personality and the FiveFactor Model: A facet-level analysis. Personality and Individual Differences, 83, 50-54.

Hough, L. M., \& Connelly, B. S. (2013). Personality measurement and use in industrial and organizational psychology. In K. F. Geisinger, B. A. Bracken, J. F. Carlson, J.-I. C. Hansen, N. R. Kuncel, S. P. Reise, \& M. C. Rodriguez (Eds.), APA handbook of testing and assessment in psychology, Vol. 1: Test theory and testing and assessment in industrial and organizational psychology (pp. 501-531). Washington, DC, US: American Psychological Association.

Hurtz, G. M., \& Donovan, J. J. (2000). Personality and job performance: The Big Five revisited. Journal of Applied Psychology, 85(6), 869-879.

Jackson, D., Paunonen, S., \& Tremblay, P. (2000). Six factor personality questionnaire manual. Port Huron, MI: Sigma Assessment Systems.

Jonason, P. K., \& O'Connor, P. J. (2017). Cutting corners at work: An individual differences perspective. Personality and Individual Differences, 107, 146-153.

Klehe, U.-C., Kleinmann, M., Hartstein, T., Melchers, K. G., König, C. J., Heslin, P. A., \& Lievens, F. (2012). Responding to personality tests in a selection context: The role of the ability to identify criteria and the ideal-employee factor. Human Performance, 25(4), 273-302.

Lee, K., \& Ashton, M. C. (2014). The dark triad, the big five, and the HEXACO model. Personality and Individual Differences, 67, 2-5.

Lee, K., Ashton, M. C., Morrison, D. L., Cordery, J., \& Dunlop, P. D. (2008). Predicting integrity with the HEXACO personality model: Use of selfand observer reports. Journal of Occupational and Organizational Psychology, 81, 147-167.

Lee, K., Gizzarone, M., \& Ashton, M. C. (2003). Personality and the likelihood to sexually harass. Sex Roles, 49, 59-69.

Loehlin, J. C., \& Goldberg, L. R. (2014). Do personality traits conform to lists or hierarchies? Personality and Individual Differences, 70, 51-56.

Markon, K. E., Krueger, R. F., \& Watson, D. (2005). Delineating the structure of normal and abnormal personality: an integrative hierarchical approach. Journal of Personality and Social Psychology, 88(1), 139157.

Markon, K. E., Quilty, L. C., Bagby, R. M., \& Krueger, R. F. (2013). The development and psychometric properties of an informant-report form of the Personality Inventory for DSM-5 (PID-5). Assessment, 20(3), 370-383.

McAbee, S. T., Oswald, F. L., \& Connelly, B. S. (2014). Bifactor models of personality and college student performance: A broad versus narrow view. European Journal of Personality, 28(6), 604-619.

McCrae, R. R. (2015). A more nuanced view of reliability: Specificity in the trait hierarchy. Personality and Social Psychology Review, 19(2), 97112. 
McCrae, R. R., \& Costa, P. T. (1987). Validation of the Five-Factor Model of personality across instruments and observers. Journal of Personality \& Social Psychology, 52, 81-90.

McCrae, R. R., \& Costa, P. T. (1995). Trait explanations in personality psychology. European Journal of Personality, 9(4), 231-252.

Morris, M. B., Burns, G. N., \& Periard, D. A. (2015). Criterion Validity of Complex Traits With Counterproductive Work Behaviors: Circumplex Versus Facet Traits. Human Performance, 28(5), 440-462.

Mõttus, R., Kandler, C., Bleidorn, W., Riemann, R., \& McCrae, R. R. (2017). Personality Traits Below Facets: The Consensual Validity, Longitudinal Stability, Heritability, and Utility of Personality Nuances. Journal of Personality and Social Psychology, 112, 474-490.

Mõttus, R., McCrae, R. R., Allik, J., \& Realo, A. (2014). Cross-rater agreement on common and specific variance of personality scales and items. Journal of Research in Personality, 52, 47-54.

Musek, J. (2007). A general factor of personality: Evidence for the Big One in the five-factor model. Journal of Research in Personality, 41(6), 12131233.

O'Neill, T., Paunonen, S., Christiansen, N., \& Tett, R. (2013). Breadth in personality assessment: Implications for the understanding and prediction of work behavior. Handbook of personality at work, 299332.

Ones, D. S., \& Viswesvaran, C. (1996). Bandwidth-fidelity dilemma in personality measurement for personnel selection. Journal of Organizational Behavior, 17(6), 609-626.

Paunonen, S. V. (1998). Hierarchical organization of personality and prediction of behavior. Journal of Personality and Social Psychology, 74, 538556.

Paunonen, S. V., \& Ashton, M. C. (2001). Big five factors and facets and the prediction of behavior. Journal of Personality and Social Psychology, 81(3), 524-539.

Paunonen, S. V., Haddock, G., Forsterling, F., \& Keinonen, M. (2003). Broad versus narrow personality measures and the prediction of behaviour across cultures. European Journal of Personality, 17(6), 413-433.

Paunonen, S. V., Rothstein, M. G., \& Jackson, D. N. (1999). Narrow reasoning about the use of broad personality measures for personnel selection. Journal of Organizational Behavior, 20(3), 389-405.

Peabody, D., \& De Raad, B. (2002). The substantive nature of psycholexical personality factors: a comparison across languages. Journal of Personality and Social Psychology, 83(4), 983-997.

Perera, H. N., Izadikhah, Z., O'Connor, P., \& McIlveen, P. (2016). Resolving dimensionality problems with WHOQOL-BREF item responses. Assessment, 1073191116678925.

Poropat, A. E. (2009). A meta-analysis of the five-factor model of personality and academic performance. Psychological Bulletin, 135(2), 322.

Revelle, W. (1995). Personality processes. Annual review of psychology, 46(1), 295-328.

Revelle, W., Condon, D. M., Wilt, J., French, J. A., Brown, A., \& Elleman, L. G. (2016). Web and phone based data collection using planned missing designs. NG Fielding.

Revelle, W., \& Wilt, J. (2013). The general factor of personality: A general critique. Journal of Research in Personality, 47(5), 493-504.

Salgado, J. F., Moscoso, S., \& Berges, A. (2013). Conscientiousness, its facets, and the prediction of job performance ratings: Evidence against the narrow measures. International Journal of Selection and Assessment, 2l(1), 74-84. 
Saucier, G. (2009). Recurrent personality dimensions in inclusive lexical studies: Indications for a Big Six structure. Journal of Personality, 77(5), 1577-1614.

Saucier, G., \& Goldberg, L. R. (2001). Lexical studies of indigenous personality factors: Premises, products, and prospects. Journal of Personality, 69(6), 847-879.

Saucier, G., Hampson, S. E., Goldberg, L. R., \& Hampson, S. E. (2000). Crosslanguage studies of lexical personality factors. Advances in personality psychology, 1, 1-36.

Saucier, G., \& Ostendorf, F. (1999). Hierarchical subcomponents of the Big Five personality factors: A cross-language replication. Journal of Personality and Social Psychology, 76(4), 613-627.

Saucier, G., \& Srivastava, S. (2015). What makes a good structural model of personality? Evaluating the Big Five and alternatives. Handbook of personality and social psychology, 3, 283-305.

Smith, M. B., Hill, A. D., Wallace, J. C., Recendes, T., \& Judge, T. A. (2018). Upsides to Dark and Downsides to Bright Personality: A Multidomain Review and Future Research Agenda. Journal of Management, 44, 191-217.

Soto, C. J., \& John, O. P. (2017). The next Big Five Inventory (BFI-2): Developing and assessing a hierarchical model with 15 facets to enhance bandwidth, fidelity, and predictive power. Journal of Personality and Social Psychology, 113(1), 117.

Steel, P., Schmidt, J., \& Shultz, J. (2008). Refining the relationship between personality and subjective well-being. Psychological Bulletin, 134(1), $138-161$.

Sun, J., Kaufman, S. B., \& Smillie, L. D. (2017). Unique associations between big five personality aspects and multiple dimensions of well-being. Journal of Personality.

Thurstone, L. L. (1934). The vectors of mind. Psychological review, 41(1), 1-32.

Veselka, L., Schermer, J. A., Petrides, K. V., Cherkas, L. F., Spector, T. D., \& Vernon, P. A. (2009). A General Factor of Personality: Evidence from the HEXACO Model and a Measure of Trait Emotional Intelligence. Twin Research and Human Genetics, 12(5), 420-424.

Wilt, J., Condon, D., \& Revelle, W. (2012). Telemetrics and online data collection. Handbook of developmental research methods, 163-179.

Wilt, J., Condon, D. M., Brown-Riddell, A., \& Revelle, W. (2012). Fundamental questions in personality. European Journal of Personality, 26(6), 629631.

Woods, S. A., \& Anderson, N. R. (2016). Toward a periodic table of personality: Mapping personality scales between the five-factor model and the circumplex model. Journal of Applied Psychology, 101(4), 582604.

Zhao, K., \& Smillie, L. D. (2015). The role of interpersonal traits in social decision making: Exploring sources of behavioral heterogeneity in economic games. Personality and Social Psychology Review, 19(3), 277-302. 